PROCEEDINGS OF THE

AMERICAN MATHEMATICAL SOCIETY

Volume 133, Number 9, Pages 2647-2656

S 0002-9939(05)08082-2

Article electronically published on April 15, 2005

\title{
A CAUCHY-SCHWARZ TYPE INEQUALITY FOR BILINEAR INTEGRALS ON POSITIVE MEASURES
}

\author{
NILS ACKERMANN
}

(Communicated by Andreas Seeger)

\begin{abstract}
If $W: \mathbb{R}^{n} \rightarrow[0, \infty]$ is Borel measurable, define for $\sigma$-finite positive Borel measures $\mu, \nu$ on $\mathbb{R}^{n}$ the bilinear integral expression

$$
I(W ; \mu, \nu):=\int_{\mathbb{R}^{n}} \int_{\mathbb{R}^{n}} W(x-y) d \mu(x) d \nu(y) .
$$

We give conditions on $W$ such that there is a constant $C \geq 0$, independent of $\mu$ and $\nu$, with

$$
I(W ; \mu, \nu) \leq C \sqrt{I(W ; \mu, \mu) I(W ; \nu, \nu)} .
$$
\end{abstract}

Our results apply to a much larger class of functions $W$ than known before.

\section{INTRODUCTION AND RESULTS}

Given a Borel function $W: \mathbb{R}^{n} \rightarrow[0, \infty]$, for $\sigma$-finite positive measures $\mu, \nu$ on $\mathbb{R}^{n}$ define the integral

$$
I(W ; \mu, \nu):=\int_{\mathbb{R}^{n}} \int_{\mathbb{R}^{n}} W(x-y) d \mu(x) d \nu(y) .
$$

Denote for $C \geq 0$ by $\mathcal{W}(n, C)$ the class of Borel functions $W: \mathbb{R}^{n} \rightarrow[0, \infty]$ such that for all $\sigma$-finite positive measures $\mu, \nu$ on $\mathbb{R}^{n}$

$$
I(W ; \mu, \nu) \leq C \sqrt{I(W ; \mu, \mu) I(W ; \nu, \nu)}
$$

holds. Moreover, denote

$$
\mathcal{W}(n):=\bigcup_{C \geq 0} \mathcal{W}(n, C) .
$$

If $W$ is an even function and the symmetric bilinear form $I(W ; \cdot, \cdot)$ is positive semidefinite, then $W \in \mathcal{W}(n, 1)$ (Cauchy-Schwarz inequality). Hence we may regard (1.1) as a generalized form of the Cauchy-Schwarz inequality.

An even function $W$ such that $I(W ; \cdot, \cdot)$ is positive semidefinite is called positive definite. Roughly speaking, positive definiteness of a function corresponds to nonnegativity of its Fourier transform [6, 5. The only result regarding (1.1) the author is aware of that goes beyond positive definite functions is given by Mattner 4. Sect. 5.1]: If $\|\cdot\|$ is any norm on $\mathbb{R}^{n}, h:[0, \infty) \rightarrow[0, \infty]$ is decreasing, and $W$ is given by $W(x):=h(\|x\|)$, then $W \in \mathcal{W}(n)$. Theorem 1.5 below recovers this

Received by the editors June 18, 2003 and, in revised form, April 21, 2004.

2000 Mathematics Subject Classification. Primary 26D15; Secondary 43A35, 35J20, 60E15.

Key words and phrases. Integral inequalities, positive definite functions, Cauchy-Schwarz inequality.

(C)2005 American Mathematical Society Reverts to public domain 28 years from publication 2647 
statement and extends it by allowing $h$ to be nonmonotone. Theorem 1.2, the main result of the present paper, yields a criterion for membership in $\mathcal{W}(n)$ for functions $W$ that cannot be written as $h \circ p$ with a seminorm $p$ on $\mathbb{R}^{n}$.

The study of property (1.1) is motivated by the partial differential equation

$$
-\Delta u+V u=\left(W * u^{2}\right) u, \quad u \in H^{1}\left(\mathbb{R}^{n}\right) .
$$

Here $*$ denotes convolution, $V$ in $L^{\infty}\left(\mathbb{R}^{n}\right)$ is periodic, and 0 lies in a gap of the spectrum of $(-\Delta+V)$; cf. [1]. One is interested in the existence of nontrivial solutions to (1.2). For the special case $n=3$ and $W(x)=1 /\|x\|_{2}$ the problem was settled in [2] by using the fact that this particular function $W$ is positive definite. In [1] it is shown that $W \in \mathcal{W}(n)$ (together with appropriate growth conditions) is sufficient to obtain a nontrivial solution of (1.2).

1.1. Main results. The statement of our theorems requires us to introduce some notation and definitions. For a topological space $X$ denote by $\mathcal{P}(X)$ the set of Borel functions $f: X \rightarrow[0, \infty]$. For $n$ in $\mathbb{N}$ denote by $\mathcal{C}(n)$ the class of subsets of $\mathbb{R}^{n}$ that are closed, convex, and symmetric (i.e. $-A=A$ ). The $\operatorname{dimension} \operatorname{dim} A$ of a convex subset $A$ of $\mathbb{R}^{n}$ is the dimension of the affine hull of $A$.

Definition 1.1. For $X, A \subseteq \mathbb{R}^{n}, X \neq \varnothing$, put

$$
\kappa(X, A):=\inf \{m \in \mathbb{N} \mid X \text { can be covered by } m \text { translates of } A\}
$$

and

$$
\alpha(X):=\inf \{m \in \mathbb{N} \mid \exists A \in \mathcal{C}(n): \operatorname{dim} A=n, A \subseteq X \text { and } \kappa(X, A)=m\} .
$$

For $X=\varnothing$ set $\kappa(\varnothing, A):=0$ and $\alpha(\varnothing):=0$.

Given a set $X$, a map $W: X \rightarrow \mathbb{R}$ and $t$ in $\mathbb{R}$ denote

$$
[W]_{t}:=\{x \in X \mid W(x) \geq t\} .
$$

Furthermore, define the class $\mathcal{A}(n)$ by

$$
\mathcal{A}(n):=\left\{W \in \mathcal{P}\left(\mathbb{R}^{n}\right) \mid \limsup _{t \rightarrow 0} \alpha\left([W]_{t}\right)+\limsup _{t \rightarrow \infty} \alpha\left([W]_{t}\right)<\infty\right\} .
$$

Our main result then reads:

Theorem 1.2. For every $n$ in $\mathbb{N}$ the inclusion $\operatorname{conv}(\mathcal{A}(n)) \subseteq \mathcal{W}(n)$ holds.

Remark 1.3. It will be shown in the proof of Theorem 1.2 that $\mathcal{W}(n)$ is a convex cone. Obviously, $\mathcal{A}(n)$ is a cone. The Example 1.6 given below demonstrates that $\mathcal{A}(n)$ is not convex.

The present author does not know whether a function in $\mathcal{W}(n)$ that is sufficiently regular, say continuous, must necessarily belong to $\operatorname{conv}(\mathcal{A}(n))$.

A simpler criterion for membership in $\mathcal{W}(n)$ can be formulated in the case of the composition of a map with a seminorm. To state it we introduce further concepts and notation.

Definition 1.4. For a subset $Y$ of $[0, \infty)$ put $\lambda(Y):=\sup \{t>0 \mid[0, t] \subseteq Y\}$ and

$$
\beta(Y):= \begin{cases}0, & Y=\varnothing, \\ \infty, & \lambda(Y)=-\infty \text { and } Y \neq \varnothing, \\ \sup (Y) / \lambda(Y), & \text { otherwise. }\end{cases}
$$

Here we set $\infty / a:=\infty$ if $a>0$ and $\infty / \infty:=1$. 
We introduce

$$
\mathcal{B}:=\left\{h \in \mathcal{P}([0, \infty)) \mid \limsup _{t \rightarrow 0} \beta\left([h]_{t}\right)+\limsup _{t \rightarrow \infty} \beta\left([h]_{t}\right)<\infty\right\} .
$$

Our second result then reads:

Theorem 1.5. Suppose that $h \in \mathcal{P}([0, \infty))$ and that $p$ is a seminorm on $\mathbb{R}^{n}$. If $h \in \mathcal{B}$, then $h \circ p \in \mathcal{A}(n)$. If $h \circ p \in \mathcal{A}(n)$ and $\operatorname{codim}(\operatorname{ker} p) \geq 2$, then $h \in \mathcal{B}$.

We provide some examples to illustrate the concepts introduced so far:

Example 1.6. Denote by $h$ the characteristic function of $[0,1]$, taken as a map from $[0, \infty)$ into $[0, \infty]$. Then $h \in \mathcal{B}$. For $i=1,2$ define $W_{i}$ as a map in $\mathcal{P}\left(\mathbb{R}^{2}\right)$ by $W_{i}\left(x_{1}, x_{2}\right):=h\left(\left|x_{i}\right|\right)$. Theorem 1.5 implies that $W_{i} \in \mathcal{A}(2)$ for $i=1,2$, but clearly $W:=W_{1}+W_{2} \notin \mathcal{A}(2)$. Since $\mathcal{A}(2)$ is a cone this implies that $\mathcal{A}(2)$ is not convex. Nevertheless, $W \in \mathcal{W}(2)$ by Theorem 1.2 and since $\mathcal{W}(2)$ is a convex cone.

Example 1.7. We construct a function $W$ in $\mathcal{A}(n)$ that is not even, and hence is neither positive definite nor of the form $h \circ p$ with $h$ in $\mathcal{P}([0, \infty))$ and $p$ a seminorm on $\mathbb{R}^{n}$. Pick $x_{0}$ in $\mathbb{R}^{n} \backslash\{0\}$ and set

$$
\begin{aligned}
W_{0}(x) & :=\frac{1}{\|x\|_{2}}, \\
W(x) & :=W_{0}(x)+W_{0}\left(x-x_{0}\right) .
\end{aligned}
$$

Denoting by $D(r, x)$ the closed ball of radius $r>0$ with center $x$, it follows easily that

$$
D(1 / t, 0) \subseteq[W]_{t} \subseteq D(2 / t, 0) \cup D\left(2 / t, x_{0}\right)
$$

for all $t>0$. This implies that $W \in \mathcal{A}(n)$.

Example 1.8. We show that the assumption on $\operatorname{codim}(\operatorname{ker} p)$ used in Theorem 1.5 is not purely technical. If $p$ is a seminorm on $\mathbb{R}^{n}$ with $\operatorname{codim}(\operatorname{ker} p)=0$, then trivially $h \circ p \in \mathcal{A}(n)$ for arbitrary $h$ in $\mathcal{P}([0, \infty))$. Given the seminorm $p(x):=|x|$ in $\mathbb{R}$ with $\operatorname{codim}(\operatorname{ker} p)=1$, we construct $h$ in $\mathcal{P}([0, \infty))$ such that $W:=h \circ p \in \mathcal{A}(1)$ but $h \notin \mathcal{B}$. Put

$$
h(s):= \begin{cases}\infty, & s=0, \\ \exp \left(-(k-1)^{2}\right), & s=\exp \left(k^{2}\right) \text { for some } k \text { in } \mathbb{N}, \\ 1 / s, & \text { otherwise. }\end{cases}
$$

For $t>1$ we obtain $[h]_{t}=[0,1 / t]$, and for $0<t \leq 1$ we obtain

$$
[h]_{t}=[0,1 / t] \cup\left\{\exp \left((1+[\sqrt{-\log t}])^{2}\right)\right\} .
$$

Recall that $[a]$ denotes the largest integer less than or equal to $a$ if $a \in \mathbb{R}$. From (1.3) it is clear that $\alpha\left([W]_{t}\right) \leq 3$ for all $t \geq 0$, so $W \in \mathcal{A}(1)$. On the other hand, for $t_{k}:=\exp \left(-k^{2}\right)$ we find

$$
\beta\left([h]_{t_{k}}\right)=\exp \left((1+k)^{2}\right) \exp \left(-k^{2}\right)=\exp (1+2 k)
$$

and therefore $\lim \sup _{t \rightarrow 0} \beta\left([h]_{t}\right)=\infty$. Hence $h \notin \mathcal{B}$. 
1.2. General notation. In $\mathbb{R}^{n}$ denote by $\|\cdot\|_{p}$ for $p$ in $[1, \infty]$ the standard $l^{p}(n)$ norm. In the case of $p=2$ we write $x \cdot y$ for the standard Euclidean scalar product of elements $x, y$ in $\mathbb{R}^{n}$. If $V$ is a subspace of $\mathbb{R}^{n}$, denote by $V^{\perp}$ the orthogonal subspace with respect to the standard scalar product.

The power set of a set $X$ will be written $2^{X}$. The cardinality of $X$ is denoted by $|X|$. Some operators used are: $\operatorname{conv} A$ for the convex hull of $A, \operatorname{cl} A, \operatorname{int} A$, and $\partial A$ for closure, interior, and boundary of a subset $A$ of a topological space.

A parallelotope is a rectangular parallelepiped.

\section{Some CONVEX GeOMETRY}

The next lemma allows us to deal with unbounded sets in $\mathcal{C}(n)$ in a convenient manner.

Lemma 2.1. If $A \in \mathcal{C}(n)$, then there is a unique subspace $V$ of $\mathbb{R}^{n}$ such that $B:=A \cap V^{\perp} \in \mathcal{C}(n)$ is compact and $A=B+V$.

Proof. First we remark: If a set $A$ in $\mathcal{C}(n)$ includes a ray (a set $\{x+t y \mid t \geq 0\}$ for some $x, y$ in $\mathbb{R}^{n}$ ), then it includes the 1-dimensional subspace parallel to that ray. If $A$ includes a translate of a subspace $V$ of $\mathbb{R}^{n}$, then $A$ includes $V$.

Now fix $A$ in $\mathcal{C}(n)$. From [3, Lemma 2.5.4] we obtain a unique subspace $V$ of $\mathbb{R}^{n}$ of maximal dimension such that a translate of $V$ and thus $V$ is included in $A$. Moreover, by that lemma it also holds that $B:=A \cap V^{\perp} \in \mathcal{C}(n)$ does not include a line (the translate of a 1-dimensional subspace) and $A=B+V$. If $B$ was not bounded, then it included a ray by 3 , Lemma 2.5.1]. Since $B$ is symmetric it therefore included a line also. Contradiction. Since $A$ is closed $B$ must therefore be compact.

If, on the other hand, for some subspace $V$ of $\mathbb{R}^{n}, B=A \cap V^{\perp}$ is compact and $A=B+V$, then $V$ is included in $A$. If $A$ includes a translate of another subspace $W$, and thus includes $W$, then $W \subseteq V$. Hence $V$ has maximal dimension among the subspaces included in $A$, and it is unique, again by Lemma 2.5.4 loc. cit.

Definition 2.2. We call the pair $(B, V)$ given for $A$ in $\mathcal{C}(n)$ by Lemma 2.1 the splitting of $A$.

Definition 2.3. Denote for $X \subseteq \mathbb{R}^{n}$ by

$$
\operatorname{ccs} X:=\operatorname{cl}\left(\operatorname{conv} \frac{1}{2}(X-X)\right) \in \mathcal{C}(n)
$$

the closed convex hull of the symmetrization of $X$.

Remark 2.4. For $A, B \subseteq \mathbb{R}^{n}$ we have $\operatorname{conv}(A+B)=\operatorname{conv} A+\operatorname{conv} B$. Thus

$$
\operatorname{ccs} X=\operatorname{cl} \frac{1}{2}(\operatorname{conv} X-\operatorname{conv} X) \text {. }
$$

From this it also follows that $\operatorname{ccs}(X+Y)=\operatorname{ccs} X+\operatorname{ccs} Y$ if one of $X$ and $Y$ is relative compact. Moreover, $\operatorname{ccs} A=A$ if $A \in \mathcal{C}(n)$.

Definition 2.5. If $X \subseteq \mathbb{R}^{n}$ and $(A, V)$ is the splitting of $\operatorname{ccs} X$, put $\gamma(X):=\operatorname{dim} V$.

Lemma 2.6. The map $\gamma: 2^{\mathbb{R}^{n}} \rightarrow\{0,1,2, \ldots, n\}$ is monotone increasing with respect to the partial order induced on $2^{\mathbb{R}^{n}}$ by inclusion. If $X \subseteq Y \subseteq \mathbb{R}^{n}$ and $\gamma(X)=\gamma(Y)$, then from $A \in \mathcal{C}(n)$ with $\operatorname{dim} A=n$ and $\kappa(X, A)<\infty$ it follows that $\kappa(Y, A)<\infty$. 
Proof. Monotonicity of $\gamma$ is obvious. Fix $X \subseteq Y$ with $\gamma(X)=\gamma(Y)$, and suppose we are given $A$ in $\mathcal{C}(n)$ with $\operatorname{dim} A=n$ and $\kappa(X, A)<\infty$. Let $(B, V)$ be the splitting of $A$ and let $\mathcal{I} \subseteq \mathbb{R}^{n}$ be finite with $X \subseteq \mathcal{I}+A=\mathcal{I}+B+V$. Since $\mathcal{I}+B$ is compact, in view of Remark 2.4 we obtain

$$
\operatorname{ccs} X \subseteq \operatorname{ccs}(\mathcal{I}+B+V)=\operatorname{ccs}(\mathcal{I}+B)+V .
$$

Since $\operatorname{ccs} X \subseteq \operatorname{ccs} Y$ and $\gamma(X)=\gamma(Y)$ there is a subspace $W$ of $\mathbb{R}^{n}$ with $\operatorname{dim} W=$ $\gamma(X)$ and there are splittings $\left(B_{1}, W\right)$ and $\left(B_{2}, W\right)$ of $\operatorname{ccs} X$ and $\operatorname{ccs} Y$, respectively, with $B_{1} \subseteq B_{2}$. Put $A_{1}:=A \cap W^{\perp}$. Now (2.1) implies $W \subseteq V$, and hence $A_{1}+W=A$. Therefore $\operatorname{dim} A=n$ yields $\operatorname{dim} A_{1}=\operatorname{dim} W^{\perp}=n-\gamma(X)$, and relint $A_{1}$ (the interior of $A_{1}$ relative to the smallest subspace including $A_{1}$ ) is open in $W^{\perp}$. Since $B_{2} \subseteq W^{\perp}$ is compact there is a finite set $\mathcal{J} \subseteq W^{\perp}$ with $B_{2} \subseteq \mathcal{J}+A_{1}$. It follows that

$$
Y \subseteq \operatorname{ccs} Y=B_{2}+W \subseteq \mathcal{J}+A_{1}+W=\mathcal{J}+A
$$

and thus $\kappa(Y, A)<\infty$.

Lemma 2.7. For all $n$ in $\mathbb{N}$ there is a constant $C_{1}(n) \geq 0$ such that for all $A$ in $\mathcal{C}(n)$ with $\operatorname{dim} A=n$ the following hold:

a) $\kappa\left(A, \frac{1}{2} A\right) \leq C_{1}(n)$,

b) there is a discrete subgroup $G$ of the additive group of $\mathbb{R}^{n}$ such that $\mathbb{R}^{n}=$ $G+A$ and $\sup _{x \in \mathbb{R}^{n}}|(x+3 A) \cap G| \leq C_{1}(n)$.

Proof. From [7, Lemma 2.4] we obtain for all $m$ in $\mathbb{N}$ a constant $C_{2}(m)$, monotone increasing in $m$, such that for every m-dimensional compact $B$ in $\mathcal{C}(m)$ there is a parallelotope $P \subseteq \mathbb{R}^{m}$, centered at the origin, with

$$
P \subseteq B \subseteq C_{2}(m) P .
$$

Now set

$$
C_{1}(n):=\left[3 C_{2}(n)+1\right]^{n},
$$

where $[a]$ denotes the largest integer below or equal to $a$ if $a \in \mathbb{R}$.

Fix $A$ in $\mathcal{C}(n)$ and let $(B, V)$ be the splitting of $A$. Since $\operatorname{dim} A=n$ we have $\operatorname{dim} B+\operatorname{dim} V=n$. We may assume $\operatorname{dim} B=m$ and $V=\{0\} \times \mathbb{R}^{n-m}$ as a subspace of $\mathbb{R}^{n}$. We identify $\mathbb{R}^{m}$ with $\mathbb{R}^{m} \times\{0\} \subseteq \mathbb{R}^{n}$ so that $B \subseteq \mathbb{R}^{m}$, and we choose a parallelotope $P \subseteq \mathbb{R}^{m}$ for $B$ as in (2.2). Then from $2 C_{2}(m) \leq 3 C_{2}(n)$ and the definition of $C_{1}(n)$ we obtain

$$
\kappa\left(A, \frac{1}{2} A\right)=\kappa\left(B, \frac{1}{2} B\right) \leq \kappa\left(C_{2}(m) P, \frac{1}{2} P\right) \leq \kappa\left(3 C_{2}(n) P, P\right) \leq C_{1}(n) .
$$

For the second assertion we use for $P$ from above the representation

$$
P=\left[-r_{1}, r_{1}\right] \times\left[-r_{2}, r_{2}\right] \times \cdots \times\left[-r_{m}, r_{m}\right]
$$

with some $r_{1}, r_{2}, \ldots, r_{m}>0$ and put $G_{0}:=2 r_{1} \mathbb{Z} \times 2 r_{2} \mathbb{Z} \times \cdots \times 2 r_{m} \mathbb{Z} \subseteq \mathbb{R}^{m}$. Then $G_{0}$ is an additive subgroup of $\mathbb{R}^{m}$ with $G_{0}+B \supseteq G_{0}+P=\mathbb{R}^{m}$. Now set $G:=G_{0} \times\{0\} \subseteq \mathbb{R}^{n}$. Then $G+A=G+B+V=\mathbb{R}^{n}$. On the other hand we have for every $x$ in $\mathbb{R}^{n}$

$$
(x+3 A) \cap G=(x+3 B) \cap G \subseteq\left(x+3 C_{2}(m) P\right) \cap G \subseteq\left(x+3 C_{2}(n) P\right) \cap G
$$

and hence

$$
|(x+3 A) \cap G| \leq\left|\left(x+3 C_{2}(n) P\right) \cap G\right| \leq C_{1}(n) .
$$

This completes the proof. 
Lemma 2.8. Suppose that $p$ is a seminorm on $\mathbb{R}^{n}$ and that $Y \subseteq[0, \infty)$. Put $X:=$ $p^{-1}(Y)$. Then $\alpha(X) \leq C_{3}(n) \beta(Y)^{n}$ for some constant $C_{3}(n)$. If $\operatorname{codim}(\operatorname{ker} p) \geq 2$, then $\alpha(X) \geq \beta(Y) / 2$.

Proof. For $r>0$ put $A(r):=\left\{x \in \mathbb{R}^{n} \mid p(x) \leq r\right\} \in \mathcal{C}(n)$. Let $(B(1), V)$ be the splitting of $A(1)$ and put $B(r):=r B(1)$ for $r>0$. Then $(B(r), V)$ is the splitting of $A(r)$. Moreover, $V=\operatorname{ker} p$. Set $m:=\operatorname{codim} V$, so $\operatorname{dim} B(1)=m$.

Define $f, g:[0, \infty) \rightarrow \mathbb{N}$ by setting $f(0):=g(0):=1$ and, for $t>0, f(t):=$ $\kappa(\partial A(t), A(1))=\kappa(\partial B(t), B(1))$ and $g(t):=\kappa(A(t), A(1))=\kappa(B(t), B(1))$. Then $f$ and $g$ are monotone increasing, $f \leq g$, and

$$
\begin{aligned}
\kappa(\partial A(r), A(s)) & =f(r / s), \\
\kappa(A(r), A(s)) & =g(r / s)
\end{aligned}
$$

for $r, s>0$. As in the beginning of the proof of Lemma 2.7 we obtain

$$
g(t)=\kappa(B(t), B(1)) \leq \kappa\left(t C_{2}(m) P, P\right)=\left[t C_{2}(m)+1\right]^{m} .
$$

Here $P \subseteq B(1)$ is a parallelotope chosen as for (2.2). If $m \geq 2$, then

$$
f(t)=\kappa(\partial B(t), B(1)) \geq t .
$$

This can be seen as follows: Consider $B(1)$ as a subset of $\mathbb{R}^{m}$. Fix $x_{0}$ in $\partial B(1)$ such that $2\left\|x_{0}\right\|_{2}=\operatorname{diam} B(1)$. Let $Q$ be the orthogonal projection in $\mathbb{R}^{m}$ onto $\operatorname{span}\left\{x_{0}\right\}$ and $L:=\operatorname{ker} Q$. Then $\operatorname{dim} L \geq 1$. It follows that for every $x$ in $\left[-t x_{0}, t x_{0}\right]$ (the segment joining $-t x_{0}$ and $\left.t x_{0}\right)$ the set $(x+L) \cap \partial B(t)$ is not empty. Moreover, from $B(1) \in \mathcal{C}(n)$ it follows that $x_{0}+L$ is a supporting hyperplane for $B(1)$. If $x_{1}, x_{2}, \ldots, x_{k} \in \mathbb{R}^{m}$ are such that

$$
\partial B(t) \subseteq \bigcup_{l=1}^{k}\left(x_{l}+B(1)\right),
$$

then from the above it is clear that

$$
\left[-t x_{0}, t x_{0}\right] \subseteq \bigcup_{l=1}^{k}\left(Q x_{l}+B(1)\right)
$$

and therefore $k \geq[t+1] \geq t$. This yields (2.4).

Let us consider the case $0<\lambda(Y) \leq \sup Y<\infty$. There is

$$
\varepsilon \in[0, \lambda(Y) / 2]
$$

such that $[0, \lambda(Y)-\varepsilon] \subseteq Y$. It follows that

$$
A(\lambda(Y)-\varepsilon) \subseteq X \subseteq A(\sup Y) .
$$

Thus, using (2.3), we obtain

$$
\alpha(X) \leq \kappa(A(\sup Y), A(\lambda(Y)-\varepsilon))=g\left(\frac{\sup Y}{\lambda(Y)-\varepsilon}\right) \leq g(2 \beta(Y)) \leq C_{3}(n) \beta(Y)^{n}
$$

for some constant $C_{3}(n) \geq 1$.

There is $\varepsilon$ in $[0, \sup Y / 2]$ such that $\sup Y-\varepsilon \in Y$ and therefore

$$
\partial A(\sup Y-\varepsilon) \subseteq X \text {. }
$$


Every $A$ in $\mathcal{C}(n)$ with $A \subseteq X$ is path connected and satisfies $0 \in A$. Since $p$ is continuous, $p(A)$ is included in the path component of $Y$ containing 0 . Therefore $p(A) \subseteq[0, \lambda(Y)]$ and $A \subseteq A(\lambda(Y))$. This shows that

$$
\kappa(X, A) \geq \kappa(X, A(\lambda(Y)))
$$

for all $A$ in $\mathcal{C}(n)$. Hence we find for $m \geq 2$, applying (2.4) and (2.5)):

$$
\alpha(X) \geq \kappa(\partial A(\sup Y-\varepsilon), A(\lambda(Y)))=f\left(\frac{\sup Y-\varepsilon}{\lambda(Y)}\right) \geq f(\beta(Y) / 2) \geq \beta(Y) / 2 .
$$

The case $\lambda(Y)>0, \sup (Y)=\infty$ is handled similarly, and in all other cases the assertion is trivial.

\section{Proof of the theorems}

Let us first prove Theorem 1.5 Suppose that we are given $h \in \mathcal{P}([0, \infty))$ and a seminorm $p$ on $\mathbb{R}^{n}$. Set $W:=h \circ p$. Then $[W]_{t}=p^{-1}\left([h]_{t}\right)$ for every $t>0$. Now Lemma 2.8 yields $\alpha\left([W]_{t}\right) \leq C \beta\left([h]_{t}\right)^{n}$ with some positive constant $C$. Moreover, if $\operatorname{codim}(\operatorname{ker} p) \geq 2$ Lemma 2.8 implies that $\beta\left([h]_{t}\right) \leq 2 \alpha\left([W]_{t}\right)$. From these facts the theorem follows.

The proof of Theorem 1.2, taken up next, is divided into the following steps:

(i) $\mathcal{W}(n, C)$ is closed under increasing pointwise limits for every $C \geq 0$.

(ii) $\mathcal{W}(n, C)$ is a convex cone for every $C \geq 0$.

Now suppose that $W \in \mathcal{P}\left(\mathbb{R}^{n}\right)$.

(iii) If $A$ in $\mathcal{C}(n)$ has dimension $n$, if $\kappa(\operatorname{supp} W, A)<\infty$, if there is $a>0$ such that $W \geq a$ on $2 A$, and if $W$ is bounded with $b:=\sup W\left(\mathbb{R}^{n}\right)$, then $W \in \mathcal{W}(n, C)$ for $C:=C_{1}(n)^{3} \kappa(\operatorname{supp} W, A) b / a$, where $C_{1}(n)$ is the constant given in Lemma 2.7.

(iv) If $\sup _{t \geq 0} \alpha\left([W]_{t}\right)<\infty$, then $W \in \mathcal{W}(n, C)$ for some $C \geq 0$.

(v) If $\lim \sup _{t \rightarrow 0} \alpha\left([W]_{t}\right)+\lim \sup _{t \rightarrow \infty} \alpha\left([W]_{t}\right)<\infty$, then $W \in \mathcal{W}(n, C)$ for some $C \geq 0$.

Theorem 1.2 is then a consequence of (ii) and (v).

Statements (i) and (ii) were proven in [4, Sect. 5.1]. For completeness we repeat the argument here. Suppose that $C \geq 0$. Fix two $\sigma$-finite positive Borel measures $\mu, \nu$ on $\mathbb{R}^{n}$. If $W$ is the pointwise limit of an increasing sequence of functions in $\mathcal{W}(n, C)$, then (1.1) follows from Lebesgue's Monotone Convergence Theorem. This proves (i) since $\mu, \nu$ were chosen arbitrarily.

Consider the implication

$$
(u \leq C \sqrt{v w} \text { and } x \leq C \sqrt{y z}) \quad \Rightarrow \quad(u+x)^{2} \leq C^{2}(v+y)(w+z)
$$

for $u, v, w, x, y, z$ in $[0, \infty)$, which is a consequence of $2 \sqrt{v w y z} \leq v z+y w$. If $W_{1}, W_{2} \in \mathcal{W}(n, C)$, then (3.1) implies that $W_{1}+W_{2} \in \mathcal{W}(n, C)$. Since $\mathcal{W}(n, C)$ is a cone, $\mathcal{W}(n, C)$ is convex.

To show (iii) choose a discrete additive subgroup $G$ of $\mathbb{R}^{n}$ for $A$ as in Lemma 2.7 b) Let $\mathcal{I}$ be a finite subset of $\mathbb{R}^{n}$ with $\operatorname{supp} W \subseteq \mathcal{I}+A$ and $|\mathcal{I}|=\kappa(\operatorname{supp} W, A)$. Put $\mathcal{J}:=(\mathcal{I}+3 A) \cap G$. From the choice of $G$ it follows that

$$
|\mathcal{J}| \leq C_{1}(n)|\mathcal{I}|
$$


Define $\bar{W}: \mathbb{R}^{n} \times \mathbb{R}^{n} \rightarrow \mathbb{R}$ by $\bar{W}(x, y):=W(x-y)$. Then $\bar{W}$ is a Borel function. We claim that

$$
\operatorname{supp} \bar{W} \subseteq \bigcup_{\substack{u, v \in G \\ u-v \in \mathcal{J}}}(u+A) \times(v+A) .
$$

To see this, suppose that $(x, y) \in \operatorname{supp} \bar{W}$, or equivalently $x-y \in \operatorname{supp} W$. There is $w$ in $\mathcal{I}$ such that $x-y \in w+A$, and there are $u, v$ in $G$ such that $x \in u+A$ and $y \in v+A$. It follows that $u-v \in x-y+2 A \subseteq w+3 A \subseteq \mathcal{I}+3 A$. Also $u-v \in G$ because $G$ is a subgroup. This proves the claim.

Now the Cauchy-Schwarz inequality for sums yields

$$
\begin{aligned}
& I(W ; \mu, \nu)=\int_{\mathbb{R}^{n} \times \mathbb{R}^{n}} \bar{W} d(\mu \times \nu) \leq b \int_{\operatorname{supp} \bar{W}} d(\mu \times \nu) \\
& \leq b \sum_{\substack{u, v \in G \\
u-v \in \mathcal{J}}} \mu(u+A) \nu(v+A) \leq b\left(\sum_{\substack{u, v \in G \\
u-v \in \mathcal{J}}} \mu(u+A)^{2} \sum_{\substack{u, v \in G \\
u-v \in \mathcal{J}}} \nu(v+A)^{2}\right)^{\frac{1}{2}} .
\end{aligned}
$$

We need to estimate the sums in the last term. For every $x$ in $\mathbb{R}^{n}$, from $A \in \mathcal{C}(n)$ it follows that the statement $(u \in G$ and $x \in u+A$ ) is equivalent to the statement $u \in(x+A) \cap G$. By the choice of $G$ this leads to

$$
|\{u \in G \mid x \in u+A\}|=|(x+A) \cap G| \leq|(x+3 A) \cap G| \leq C_{1}(n)
$$

and thus for all $x, y$ in $\mathbb{R}^{n}$

$$
|\{u \in G \mid(x, y) \in(u+A) \times(u+A)\}| \leq C_{1}(n)^{2} .
$$

We also have

$$
\bigcup_{u \in G}(u+A) \times(u+A) \subseteq\left\{(x, y) \in \mathbb{R}^{n} \times \mathbb{R}^{n} \mid x-y \in 2 A\right\}=: D
$$

and $\bar{W} \geq a$ on $D$. Using (3.2), (3.4) and (3.5) we calculate

$$
\begin{array}{r}
\sum_{\substack{u, v \in G \\
u-v \in \mathcal{J}}} \mu(u+A)^{2}=|\mathcal{J}| \sum_{u \in G} \mu(u+A)^{2}=|\mathcal{J}| \sum_{u \in G} \int_{(u+A) \times(u+A)} d(\mu \times \mu) \\
\leq C_{1}(n)^{2}|\mathcal{J}| \int_{D} d(\mu \times \mu) \leq \frac{C_{1}(n)^{3}|\mathcal{I}|}{a} \int_{\mathbb{R}^{n} \times \mathbb{R}^{n}} \bar{W} d(\mu \times \mu) \\
=\frac{C_{1}(n)^{3}|\mathcal{I}|}{a} I(W ; \mu, \mu),
\end{array}
$$

a similar estimate holding for the sum over $\nu(v+A)^{2}$. This proves (iii) in view of (3.3).

To show (iv) suppose that $M:=\sup _{t \geq 0} \alpha\left([W]_{t}\right)<\infty$. For $m$ in $\mathbb{N}$ and $1 \leq i \leq$ $m 2^{m}$ define $W_{m, i}$ and $W_{m}$ in $\mathcal{P}\left(\mathbb{R}^{n}\right)$ by setting

$$
\begin{aligned}
W_{m, i} & :=\frac{1}{2^{m}} \chi_{[W]_{i / 2^{m}}}, \\
W_{m} & :=\sum_{i=1}^{m 2^{m}} W_{m, i} .
\end{aligned}
$$


Here $\chi_{A}$ denotes for $A \subseteq \mathbb{R}^{n}$ the characteristic function of $A$. The sequence $\left(W_{m}\right)$ is increasing and converges pointwise to $W$. Fix $m$ and $i$. There is $A$ in $\mathcal{C}(n)$ such that $\operatorname{dim} A=n, A \subseteq[W]_{i / 2^{m}}$, and $\kappa\left([W]_{i / 2^{m}}, A\right) \leq M$. Since $A$ is closed $\operatorname{supp} W_{m, i}=\operatorname{cl}[W]_{i / 2^{m}}$ can be covered by the same number of translates of $A$ as $[W]_{i / 2^{m}}$, i.e. $\kappa\left(\operatorname{supp} W_{m, i}, A\right)=\kappa\left([W]_{i / 2^{m}}, A\right)$. Using Lemma 2.7 we thus obtain

$$
\kappa\left(\operatorname{supp} W_{m, i}, \frac{1}{2} A\right) \leq C_{1}(n) \kappa\left(\operatorname{supp} W_{m, i}, A\right) \leq C_{1}(n) M .
$$

Moreover, $W_{m, i}=1 / 2^{m}$ on $A$ and $W_{m, i} \leq 1 / 2^{m}$ on $\mathbb{R}^{n}$. By (iii) $W_{m, i} \in \mathcal{W}(n, C)$ for $C=C_{1}(n)^{4} M$, independently of $m$ and $i$. By (ii) $W_{m} \in \mathcal{W}(n, C)$ for every $m$, and thus (i) yields the desired result.

The remaining case (v) is handled as follows: We can assume that $W \neq 0$, otherwise there is nothing to do. By our assumptions there are $M>0$ and $0<$ $t_{1}<t_{0}$ such that $\alpha\left([W]_{t}\right) \leq M$ for $t$ in $\left(0, t_{1}\right] \cup\left[t_{0}, \infty\right)$ and $[W]_{t} \neq \varnothing$ for $t$ in $\left(0, t_{1}\right]$. Consider $\gamma\left([W]_{t}\right)$ as a function of $t$ sending $(0, \infty)$ into $\{0,1,2, \ldots, n\}$ ( $\gamma$ is given in Definition [2.5). We can choose $0<t_{3}<t_{2} \leq t_{1}$ with $\gamma\left([W]_{t_{2}}\right)=\gamma\left([W]_{t_{3}}\right)$. For $x$ in $\mathbb{R}^{n}$ put $W_{1}(x):=\min \left\{t_{3}, W(x)\right\}$ and $W_{2}(x):=\min \left\{t_{0}-t_{3}, W(x)-W_{1}(x)\right\}$. Also put $W_{3}:=W-W_{1}-W_{2}$. Then $W_{1} \leq t_{3}, W_{2} \leq t_{0}-t_{3}$, and $W_{i} \geq 0$ for $i=1,2,3$. Moreover, we have

$$
\begin{aligned}
{\left[W_{1}\right]_{t} } & = \begin{cases}{[W]_{t},} & 0 \leq t \leq t_{3}, \\
\varnothing, & t_{3}<t,\end{cases} \\
{\left[W_{2}\right]_{t} } & = \begin{cases}{[W]_{t+t_{3}},} & 0 \leq t \leq t_{0}-t_{3}, \\
\varnothing, & t_{0}-t_{3}<t,\end{cases} \\
{\left[W_{3}\right]_{t} } & =[W]_{t+t_{0}} .
\end{aligned}
$$

From (iv) it follows that $W_{1}, W_{3} \in \mathcal{W}(n, C)$ for some $C \geq 0$. Since $[W]_{t_{2}} \neq \varnothing$ and $\alpha\left([W]_{t_{2}}\right)<\infty$ there is $A$ in $\mathcal{C}(n)$ with $\operatorname{dim} A=n, A \subseteq[W]_{t_{2}}$ and $\kappa\left([W]_{t_{2}}, A\right)<\infty$. By Lemma 2.6 $\kappa\left([W]_{t_{3}}, A\right)<\infty$ also, and by Lemma 2.7 $\left.{ }^{2}\right) \kappa\left([W]_{t_{3}}, \frac{1}{2} A\right)<\infty$. Hence the closedness of $A$ and supp $W_{2} \subseteq \operatorname{cl}[W]_{t_{3}}$ imply that $\kappa\left(\operatorname{supp} W_{2}, \frac{1}{2} A\right)<\infty$. We also have $W_{2} \geq t_{2}-t_{3}$ on $A$ and $W_{2} \leq t_{0}-t_{3}$ on $\mathbb{R}^{n}$. Now (iii) implies that $W_{2} \in \mathcal{W}(n, C)$ for some $C$, and by (ii) the same holds for $W=W_{1}+W_{2}+W_{3}$. This finishes the proof of (v).

\section{REFERENCES}

[1] N. Ackermann, On a periodic Schrödinger equation with nonlocal superlinear part, Math. Z. 248 (2004), no. 2, 423-443. MR2088936

[2] B. Buffoni, L. Jeanjean, and C.A. Stuart, Existence of a nontrivial solution to a strongly indefinite semilinear equation, Proc. Amer. Math. Soc. 119 (1993), no. 1, 179-186. MR 1145940 (93k:35086)

[3] B. Grünbaum, Convex polytopes, With the cooperation of Victor Klee, M. A. Perles and G. C. Shephard. Pure and Applied Mathematics, Vol. 16, Interscience Publishers, John Wiley \& Sons, Inc., New York, 1967. MR0226496|(37:2085)

[4] L. Mattner, Strict definiteness of integrals via complete monotonicity of derivatives, Trans. Amer. Math. Soc. 349 (1997), no. 8, 3321-3342. MR1422615 (97m:26026)

[5] Z. Sasvári, Positive definite and definitizable functions, Mathematical Topics, vol. 2, Akademie Verlag, Berlin, 1994. MR.1270018 (95c:43005) 
[6] J. Stewart, Positive definite functions and generalizations, an historical survey, Rocky Mountain J. Math. 6 (1976), no. 3, 409-434. MR0430674 (55:3679)

[7] C. Zong, Strange phenomena in convex and discrete geometry, Universitext, Springer-Verlag, New York, 1996. MR1416567 (97m:52001)

Justus-Liebig-Universität, Mathematisches Institut, Arndtstr. 2, D-35392 Giessen, Germany

E-mail address: nils.ackermann@math.uni-giessen.de 